\title{
ELABORAÇÃO DE MODELO DE PREVISÃo DE PREÇO DA MADEIRA DE Eucalyptus spp.
}

\author{
Naisy Silva Soares ${ }^{1}$, Márcio Lopes da Silva², José Luiz Pereira de Rezende ${ }^{3}$, \\ João Eustáquio de Lima ${ }^{4}$, Kaio Henrique Adame de Carvalho ${ }^{5}$
}

(recebido: 17 de outubro de 2008; aceito: 27 de novembro de 2009)

RESUMO: Conduziu-se este trabalho com o objetivo de elaborar um modelo para prever o preço da madeira em pé de Eucalyptus spp. em Itapeva (SP) e Bauru (SP), utilizando a metodologia de Box \& Jenkins (1976) em observações mensais, cobrindo o período de maio de 2002 a janeiro de 2008. Os resultados obtidos indicaram que o modelo adequado para efetuar as previsões do preço da madeira de Eucalyptus spp. em Itapeva e Bauru foram, respectivamente, um ARIMA $(0,1,4)$ e um ARIMA $(2,1,2)$.

Palavras-chave: Séries temporais, modelo ARIMA, previsão de preços.

\section{Eucalyptus spp. WOOD PRICE FORECASTING MODEL}

ABSTRACT: The objective of this paper was to establish a price forecasting model of Eucalyptus spp. wood in Itapeva (SP) and Bauru (SP), using Box \& Jenkins (1976) methodology in monthly observations, from May 2002 to January 2008. The results shows that the appropriate models for forecasting Eucalyptus spp. wood price in Itapeva $(S P)$ and Bauru $(S P)$ were, respectively, ARIMA $(0,1,4)$ and ARIMA $(2,1,2)$.

Key words: Times series, ARIMA models, price forecasting, Eucalyptus spp. wood.

\section{INTRODUÇÃO}

O Eucalyptus spp. é um gênero arbóreo nativo da Austrália, com mais de 600 espécies conhecidas. A introdução do Eucalyptus spp., em bases técnicas no Brasil, iniciou-se em 1904, no Horto de Jundiaí (SP), conduzida por Edmundo Navarro de Andrade. Entretanto, há informações de existência de dois exemplares de $E$. gigantea no Jardim Botânico do Rio de Janeiro, em 1825, e de alguns exemplares no Rio Grande do Sul, em 1865 (ASSOCIAÇÃO MINEIRA DE SILVICULTURA - AMS, 2008).

A área plantada total com o gênero no país atingiu 3.351.867 ha em 2007, sendo Minas Gerais o Estado que contribuiu com o maior percentual (28\%), seguido por São Paulo (22\%), Bahia (15\%) e Espírito Santo (6\%) (ASSOCIAÇÃO BRASILEIRA DE PRODUTORES DE FLORESTAS PLANTADAS - ABRAF, 2008).

Em 2006, a produtividade média das florestas nacionais de Eucalyptus spp. alcançou $40 \mathrm{~m} 3 / \mathrm{ha} / \mathrm{ano}$. Em termos comparativos, no Uruguai, Indonésia, Chile, Estados
Unidos, Canadá, Espanha e Finlândia, a produtividade média das florestas de Eucalyptus spp., naquele ano, foi 25, 20, 25, 10, 7, 10 e 4 mª/hano, respectivamente. Além disso, no Brasil a rotação florestal é de 7 anos, inferior a de países como África do Sul (8-10 anos), Chile (10-12 anos), Portugal (12-15 anos), Espanha (12-15 anos) (ASSOCIAÇÃO BRASILEIRA DE CELULOSE E PAPEL-BRACELPA, 2007; VOTORANTIM CELULOSE E PAPEL - VCP, 2004).

A importância desse gênero pode ser atribuída à sua múltipla utilização e ao seu consumo crescente. Em 2005, o consumo da madeira de Eucalyptus spp. no Brasil concentrou-se, principalmente, na produção de celulose e na siderurgia a carvão vegetal, segmentos que consomem entre $65 \%$ e $75 \%$ do total (AMS, 2008).

Segundo Soares (2006), o consumo da madeira de Eucalyptus spp. pelas empresas de celulose no país cresceu, em média, 5,8\% ao ano, de 1980 a 2005. Gomes (2006) verificou que o consumo dessa matéria-prima nas empresas de ferro-gusa de Minas Gerais cresceu, aproximadamente, $22 \%$, no período de 2001 a 2005 ,

\footnotetext{
${ }^{1}$ Economista, Doutoranda em Ciência Florestal - Departamento de Engenharia Florestal/DEF - Universidade Federal de Viçosa/UFV Avenida Peter Henry Rolfs, s/n - Campus Universitário - 36570-000 - Viçosa, MG - naisysilva@yahoo.com.br

${ }^{2}$ Engenheiro Florestal, Professor Dr. em Economia Florestal - Departamento de Engenharia Florestal/DEF - Universidade Federal de Viçosa/UFV - Avenida Peter Henry Rolfs, s/n - Campus Universitário - 36570-000 - Viçosa, MG - marlosil@ufv.br

${ }^{3}$ Engenheiro Florestal, Professor Dr. em Economia Florestal - Departamento de Ciências Florestais/DCF - Universidade Federal de Lavras/UFLA - Cx. P. 3037 - 37200-000 - Lavras, MG - jlprezen@dcf.ufla.br

${ }^{4}$ Engenheiro Agrônomo, Professor Dr. em Economia Rural - Departamento de Economia Rural/DER - Universidade Federal de Viçosa/ UFV - Avenida Peter Henry Rolfs, s/n - Campus Universitário - 36570-000 - Viçosa, MG - jelima@ufv.br

${ }^{5}$ Engenheiro Florestal, Mestrando em Ciência Florestal - Departamento de Engenharia Florestal/DEF - Universidade Federal de Viçosa/ UFV - Avenida Peter Henry Rolfs, s/n - Campus Universitário - 36570-000 - Viçosa, MG - kaio_adame@ hotmail.com
}

Cerne, Lavras, v. 16, n. 1, p. 41-52, jan./mar. 2010 
enquanto outros segmentos como lenha, madeira serrada, painéis, construções rurais, postes de eletrificação e estacas de cerca também estão utilizando de forma crescente a madeira de Eucalyptus spp. (AMS, 2008).

Pode-se, portanto, afirmar que a madeira de Eucalyptus spp. a cada dia se torna mais importante, tanto para produtores (por uma alternativa produtiva) como para insumo industrial.

Assim, pesquisas que busquem estimar modelos para previsão dos preços da madeira de Eucalyptus spp. no Brasil são importantes para os produtores por fornecer orientações sobre os melhores meios de selecionar seus empreendimentos, dimensionar suas vendas e planejar o volume de estoques. Para o governo também é importante, pois pode subsidiar o planejamento de políticas para o desenvolvimento do mercado da madeira de Eucalyptus spp. no país (SILVA \& SILVA, 1996).

Em síntese, a previsão do preço da madeira de Eucalyptus spp. no Brasil deve ser uma ferramenta de apoio a tomadas de decisões futuras, de eficiência comprovada, precisão de resultados, simplicidade nos métodos empregados e confiabilidade estatística do modelo.

No entanto, grande parte da madeira de Eucalyptus spp. no país é comercializada entre regiões próximas. Em 2006, por exemplo, $50 \%$ dessa madeira foi transportada por até $100 \mathrm{~km}, 19 \%$ de 101 a $200 \mathrm{~km}, 12 \%$ de 201 a $300 \mathrm{~km}, 17 \%$ de 301 a $400 \mathrm{~km}$ e $2 \%$ de 402 a $500 \mathrm{~km}$ (BRACELPA, 2006). Como a madeira de reflorestamento em tora apresenta elevado peso e baixo preço, o custo de transporte é elevado (SILVA et al., 2007; VALVERDE et al., 2005), explicando a pouca expressividade do comércio da madeira de Eucalyptus spp. entre regiões distantes.

Sendo assim, acredita-se que alterações nos preços da madeira de Eucalyptus spp. em tora em um mercado não são transmitidas aos preços de outros mercados quando o preço for baixo a ponto de não compensar transportá-la por longas distâncias. Ou seja, conforme Basu (2006), os mercados não são integrados espacialmente. Consequentemente, um modelo de previsão de preços da madeira de Eucalyptus spp. para uma região não deve ser válido para outras regiões.

Nesse contexto, em termos gerais, o presente trabalho buscou elaborar um modelo para prever o preço da madeira de Eucalyptus spp. Mais especificamente, pretendeu-se estimar um modelo que permita prever os preços da árvore em pé de Eucalyptus spp. em duas regiões do Brasil, Itapeva (SP) e Bauru (SP), com base em técnicas estatísticas de previsão.

Cerne, Lavras, v. 16, n. 1, p. 41-52, jan./mar. 2010

\section{MATERIAL E MÉTODOS}

\subsection{Referencial Teórico}

Para fornecer respostas a este estudo, tomou-se como referência a metodologia desenvolvida por Box \& Jenkins (1976) que consiste em quatro etapas, a saber:

i) Identificação: Compreende um conjunto de procedimentos com o objetivo de proporcionar uma idéia apropriada da estrutura do modelo (FISHER, 1982).

Para Fisher (1982), inicialmente, é conveniente plotar os valores da série observada. Seu gráfico possibilita a visualização de uma série com tendência crescente, geométrica, sugerindo a inexistência de componente sazonal significativa. Por outro lado, pode sugerir a possibilidade de conveniência de transformação da variável para estabilizar a variância. de modelos.

Segundo Rocha (2003), tem-se as seguintes classes

- Média Móvel (MA): No modelo MA a série temporal $Y_{t}$ é resultado da combinação linear dos termos aleatórios em $t$ e em períodos anteriores. Em sua forma geral ou um MA(q), leva em consideração $q$ valores defasados de $\varepsilon$ (equação 1):

$Y_{t}=\varepsilon_{t}-\theta_{1} \varepsilon_{t-1}-\theta_{2} \varepsilon_{t-2} \ldots \ldots \ldots \theta_{p} \varepsilon_{t-p}$

em que: $\theta=$ parâmetro; e $\varepsilon_{t}=$ termos aleatórios.

- Auto-regressivo (AR): No modelo AR, a série temporal $Y_{t}$ é descrita somente pelos seus valores realizados e pelos termos aleatórios. $\mathrm{O}$ referido modelo de ordem $p \mathrm{AR}(\mathrm{p})$ será o resultado da soma ponderada de seus $p$ valores passados e dos termos aleatórios (equação 2):

$Y_{t}=\phi_{1} Y_{t-1}+\phi_{2} Y_{t-2}+\ldots \ldots \ldots+\phi_{p} Y_{t-p}+\varepsilon_{t}$

em que: $\phi_{t}=$ parâmetro; e $\varepsilon_{t}=$ termos aleatórios.

- Média Móvel Auto-regressivo (ARMA): No modelo ARMA, a série temporal $Y_{t}$ é função de seus valores históricos e pelos termos aleatórios correntes e passados. Generalizando, este modelo é representado como (equação 3):

$$
Y_{t}=\phi_{1} Y_{t-1}+\ldots \ldots+\phi_{p} Y_{t-p}+\varepsilon_{t}-\theta_{1} \varepsilon_{t-1}-\ldots \ldots-\theta_{p} \varepsilon_{t-p}
$$

- Média Móvel Auto-regressivo e Integrado (ARIMA): Se a série temporal analisada não for estacionária em nível, devem-se aplicar diferenças até que a condição de estacionariedade se torne válida. $\mathrm{O}$ número de diferenças necessárias é denominado ordem de integração (I). Sendo $Y_{t}$ estacionária, após ser diferenciada $d$ vezes, com a série 
derivada desse processo, podendo ser descrita como um modelo ARMA(p,q), diz-se que a série temporal $Y_{t}$ segue um modelo $\operatorname{ARIMA}(p, d, q)$ (equação 4).

$$
W_{t}=\phi_{1} W_{t-1}+\ldots \ldots+\phi_{p} W_{t-p}+\varepsilon_{t}-\theta_{1} \varepsilon_{t-1}-\ldots \ldots-\theta_{q} \varepsilon_{t-q}
$$

em que: $W_{t}=\Delta^{d} Y_{t}$

- Modelos Sazonais:

- Modelo sazonal auto-regressivo (SAR(P)): O Modelo $\mathrm{SAR}(\mathrm{P})$ corresponde à versão sazonal do $\mathrm{AR}(\mathrm{p})$ (equação 5):

$$
Y_{t}=\Phi_{1} Y_{t-S}+\Phi_{2} Y_{t-2 S}+\ldots \ldots \ldots+\Phi_{p} Y_{t-p S}+\varepsilon_{t}
$$

- Modelo sazonal de média móvel (SMA(Q)): O modelo $\operatorname{SMA}(\mathrm{Q})$ é equivalente à versão sazonal do MA(q) (equação 6):

$$
Y_{t}=\varepsilon_{t}-\Theta_{1} \varepsilon_{t-s}-\Theta_{2} \varepsilon_{t-2 S}-\ldots \ldots . . \Theta_{Q} \varepsilon_{t-Q S}
$$

- Modelo sazonal autorregressivo de média móvel (SARMA(P,Q)): O Modelo SARMA(P,Q) é a versão sazonal do $\operatorname{ARMA}(p, q)$ (equação 7):

$$
Y_{t}=\Phi_{1} Y_{t-S}+\Phi_{2} Y_{t-2 S}+\ldots \ldots+\Phi_{P} Y_{t-P S}+\varepsilon_{t}-\Phi_{1} \varepsilon_{t-S}-\ldots \ldots-\Phi_{Q} \varepsilon_{t-Q S}
$$

- Modelo sazonal auto-regressivo integrado de média móvel (SARIMA(P,D,Q)): O SARIMA(P,D,Q) dedicase a séries originais não estacionárias, que após $\mathrm{D}$ diferenças sazonais podem ser descritas por um processo estocástico sazonal estacionário SARMA(P,Q). O referido modelo em sua forma generalizada pode ser representado como (equação 8):

$$
W_{t}=\Phi_{1} W_{t-S}+\ldots \ldots+\Phi_{p} W_{t-p S}+\varepsilon_{t}-\Phi_{1} \varepsilon_{-S}-\ldots \ldots-\Phi_{Q} \varepsilon_{t-Q S}
$$

Quando se considera, em conjunto, as correlações temporais entre observações em períodos de tempo sucessivos, encontra-se o modelo sazonal multiplicativo geral ARIMA(p,d,q)x(P,D,Q) que combina os modelos ARIMA sazonal e não sazonal.

Para cada um desses modelos, a ordem pode variar. Deve-se optar por modelos de ordem 1 ou 2, com base no "principio de parcimônia", isto é, deve-se obter um modelo mais simples com um pequeno número de parâmetros (SILVA \& SILVA, 1996).

As principais ferramentas da identificação são a função de autocorrelação (FAC) (equação 9), a função de autocorrelação parcial (FACP) (equação 10) e os correlogramas resultantes, que são as representações gráficas das FACs e FACPs (ENDERS, 1995; GUJARATI, 2000). $\rho_{k}=\frac{\gamma_{k}}{\gamma_{0}}$

em que: $\rho_{k}=$ coeficiente de autocorrelação na defasagem $k ; \gamma_{k}=$ covariância na defasagem $k ; \gamma_{0}=$ variância.

$\hat{\gamma}_{k}=\frac{\sum\left(Y_{t}-\bar{Y}\right)\left(Y_{t+k}-\bar{Y}\right)}{n}=\hat{\gamma}_{0}=\frac{\sum\left(Y_{t}-\bar{Y}\right)^{2}}{n}=\hat{\rho}=\frac{\hat{\gamma_{k}}}{\hat{\gamma}_{o}}$

em que: $\hat{\gamma}_{k}=$ covariância amostral na defasagem $k ; \hat{\gamma}_{0}=$ variância amostral; $\hat{\rho}_{k}=$ função de autocorelação amostral. O intervalo de confiança de $95 \%$ para qualquer $\hat{\rho}_{k}$, seguindo uma distribuição normal padrão é dado por (equação 11):

$I C= \pm 1,96 * \frac{1}{n}$

em que: IC $=$ intervalo de confiança; $\frac{1}{n}=$ variância; e $n=$
número de observações. Se um $\hat{\rho}_{k}$ se localizar dentro do intervalo de confiança, podemos aceitar a hipótese de que o verdadeiro valor de $\rho_{k}$ é zero e vice-versa.

A estatística Q de Box e Pierce pode ser utilizada para testar a hipótese conjunta de que todos $\rho_{k}$ são simultaneamente iguais a zero. Essa estatística se distribui aproximadamente como a distribuição Qui-quadrado com $m$ graus de liberdade e é definida como (equação 12):

$Q=n \sum_{k=1}^{m} \hat{\rho_{k}^{2}}$

em que: $n=$ número de observações; e $m=$ duração da defasagem.

ii) Estimação: Após a identificação do modelo apropriado, a próxima etapa é estimar os parâmetros dos termos autorregressivos e de média móvel incluídos no modelo.

iii) Avaliação: A avaliação consiste em checar se o modelo escolhido é adequado para os fins desejados. Isso pode ser verificado pelo comportamento da FAC e da FACP dos resíduos do modelo estimados, e por meio do teste de $\chi^{2}$ de Box-Pierce, que compara o valor da estatística de teste com os valores tabelados da distribuição Qui-quadrado com $k-p-q$ graus de liberdade. Para que o modelo estimado seja considerado adequado é necessário que os seus resíduos sejam estimativas do ruído branco, ou seja, suas autocorrelações devem se comportar de maneira aleatória e devem ser não significativas (GUJARATI, 2000).

Cerne, Lavras, v. 16, n. 1, p. 41-52, jan./mar. 2010 
iv) Previsão: Nesta etapa realiza-se a checagem da confiabilidade da previsão do modelo. Para se obter melhores resultados na utilização da metodologia de Box \& Jenkins (1976), a amostra deve ser de no mínimo 50 observações; a série deve ser estacionária, ou seja, a série deve variar em torno de uma média e de uma variância constante; e a série deve ser homocedástica, ou seja, deve possuir uma variância constante ao longo do tempo (SANTOS \& LIMA, 2006).

$\mathrm{Na}$ área florestal, a metodologia de Box \& Jenkins (1976) já foi utilizada em alguns trabalhos para previsão de preços.

Silva \& Silva (1996), por exemplo, analisando o comportamento dos preços do carvão vegetal, em Minas Gerais, no período de 1980 a 1992, constataram que o modelo ARIMA $(1,1,1)$ forneceu boas estimativas e boa previsão para os preços da referida matéria-prima.

Coelho Junior et al. (2006a), analisaram os preços do carvão vegetal, em Minas Gerais, de janeiro de 1975 a dezembro de 2002. Foi observado que o modelo SARIMA $(2,0,1)(0,1,1)_{12}$ proporcionou melhor ajuste para previsão dos preços do carvão vegetal, no Estado.

Coelho Junior et al. (2006b), verificaram que, de janeiro de 1999 a abril de 2004, o SARIMA $(2,1,2)(0,1,0)_{12}$ e o SARIMA $(0,1,2)(0,1,0)_{12}$ proporcionaram melhor ajuste para previsão dos preços do carvão de origem plantada e de origem nativa, respectivamente, em Minas Gerais.

Soares et al. (2008), constataram que o modelo ARIMA $(2,1,1)$ forneceu boas estimativas para a previsão dos preço da borracha natural no Brasil, de janeiro de 1999 a setembro de 2007.

Coelho Junior et al. (2009) também estimaram um modelo para a previsão dos preços da borracha natural no Brasil. Porém, considerando o período de janeiro de 1999 a junho de 2006. Os autores observaram que o modelo ARIMA $(1,1,1)$ proporcionou melhor ajuste.

Oliveira et al. (1977) utilizaram dados semanais de janeiro de 1973 a dezembro de 1975 para a previsão do preço futuro de diferentes tipos de madeira serrada nos Estados Unidos. O modelo ARIMA foi eficaz na previsão do preço da madeira serrada para os dados utilizados.

Song (2003) utilizou a metodologia de Box \& Jenkins (1976) para prever o preço da madeira serrada nos Estados Unidos, de janeiro de 1990 a dezembro de 2003. O autor concluiu que o melhor modelo para previsão do preço da madeira serrada foi o $\operatorname{ARIMA}(13,1,0)$.

Cerne, Lavras, v. 16, n. 1, p. 41-52, jan./mar. 2010

\subsection{Referencial Analítico}

A seguir estão os procedimentos para determinar um modelo de previsão de preços da madeira de Eucalyptus spp. em Itapeva (SP) e Bauru (SP) com base na metodologia de Box \& Jenkins (1976).

i) Identificação: A ordem $d$ do modelo foi verificada pelo teste de raiz unitária utilizando a metodologia de Dickey-Fuller Aumentado (ADF) para determinar a ordem de integração das variáveis consideradas. O referido teste, nas séries sob análise, foi aplicado nas seguintes formas, conforme Seddighi et al. (2000):

- sem constante e sem tendência:

$\Delta X_{t}=\delta X_{t-1}+\sum_{i=1}^{m} \delta_{i} \Delta X_{t-i}+u_{t}$

- com constante e sem tendência:

$\Delta X_{t}=\beta_{1}+\delta X_{t-1}+\sum_{i=1}^{m} \delta_{i} \Delta X_{t-i}+u_{t}$

- com constante e com tendência:

$\Delta X_{t}=\beta_{1}+\beta_{2} t+\delta X_{t-1}+\sum_{i=1}^{m} \delta_{i} \Delta X_{t-i}+u_{t}$

em que: $\Delta_{t}=$ operador de diferença, $X=$ variável dependente, $\delta=$ raiz associada à variável dependente defasada, $\beta$ são parâmetros a serem estimados.

As hipóteses testadas são: $|\tau|_{\text {calculado }}>|\tau|_{\text {critico }}$ :rejeita-se $H_{0}: \delta=0$, e a série é estacionária; e $|\tau|_{\text {calculado }}<|\tau|_{\text {crítico }}$ : aceita-se $H_{0}: \delta=0$, e a série é não-estacionária.

A ordem $p$ e $q$ do modelo e a sazonalidade foi analisada com base na FACP e na FAC.

ii) Estimação: Com base na análise da FAC e da FACP estimou-se o modelo de previsão de preços da madeira de Eucalyptus spp. no Brasil, pelo método Mínimos Quadrados Ordinários (MQO).

iii) Avaliação: Para avaliar o modelo estimado analisou-se o correlograma e os resultados do teste ADF nos resíduos do modelo.

iv) Previsão: Depois de estimado e avaliado foram realizadas 12 previsões para o modelo escolhido para visualizar melhor o poder preditivo do mesmo.

Foi calculado o erro percentual (Erro (\%)), conforme equação (16), no sentido de quantificar o desvio entre o preço observado e o previsto.

$\operatorname{Erro}(\%)=\left[\left(\frac{\text { Preço previsto }}{\text { Preçoobservado }}\right)-1\right] * 100$ 


\subsection{Fonte de dados}

Os dados utilizados referem-se aos preços médios mensais da árvore em pé de Eucalyptus spp. nas praças de Itapeva (SP) e Bauru (SP), no período de maio de 2002 a janeiro de 2008. Esses preços estão em R \$/st e são do Centro de Estudos Avançados em Economia Aplicada CEPEA (CEPEA, 2008). O tamanho da amostra, a decisão de utilizar dados mensais e a escolha das regiões para análise foi definida em função da disponibilidade de dados.

Ressalta-se que nas estimativas foram deixadas de fora as doze últimas observações com objetivo de fazer uma previsão prévia para melhor avaliar o modelo, e que se trabalhou com preços nominais, pois no período considerado para análise, a inflação foi baixa e apresentou tendência bem definida. Sendo assim, as estimativas obtidas a partir de preços correntes são muito semelhantes às obtidas utilizando preços reais, portanto, é dispensável calcular previamente os preços reais (CÉZAR et al., 1995).

\section{RESULTADOS E DISCUSSÃO}

Os resultados obtidos, por meio da metodologia de Box \& Jenkins (1976), estão a seguir.

i) Identificação:

Conforme sugerido por Fischer (1982), na Figura 1, observa-se o esboço do gráfico com a série mensal de preço da madeira de Eucalyptus spp. (R $\$ /$ st), no período de maio de 2002 a janeiro de 2008.
Como mostra a Figura 1, os preços nominais da madeira de Eucalyptus spp. aumentaram, no período de maio de 2002 a janeiro de 2008. O aquecimento da demanda e a estabilização da área plantada podem explicar esse aumento dos preços, conforme observou Valverde et al (2006).

Com a análise gráfica preliminar, pode-se inferir existência de tendência crescente, inexistência de sazonalidade e não estacionariedade da série de preços da madeira de Eucalyptus spp..

Os resultados do teste ADF confirmam que as séries de preços, sob análise, não são estacionárias em nível, tendo em vista que os valores calculados são menores em módulo que seus respectivos valores críticos em todos os modelos analisados. Mas, na primeira diferença as séries de preços da madeira de Eucalyptus spp. tornaram-se estacionárias (Tabela 1).

Para a série de preços de Itapeva, na primeira diferença, observou-se na FAC um pico no lag 15 indicando a inclusão do MA(15). Na FACP não foram observados picos fora do intervalo de confiança (Figura 2).

Na série de preços da madeira de Eucalyptus spp., em Bauru, na primeira diferença, verificou-se dois picos fora do intervalo de confiança nos lags 7 e 13 na FACP, sugerindo a inclusão do $\operatorname{AR}(7)$ e $\operatorname{AR}(13)$. Na FAC, foi observado o mesmo. Assim, tem-se o MA(7) e o MA(13) (Figura 3).

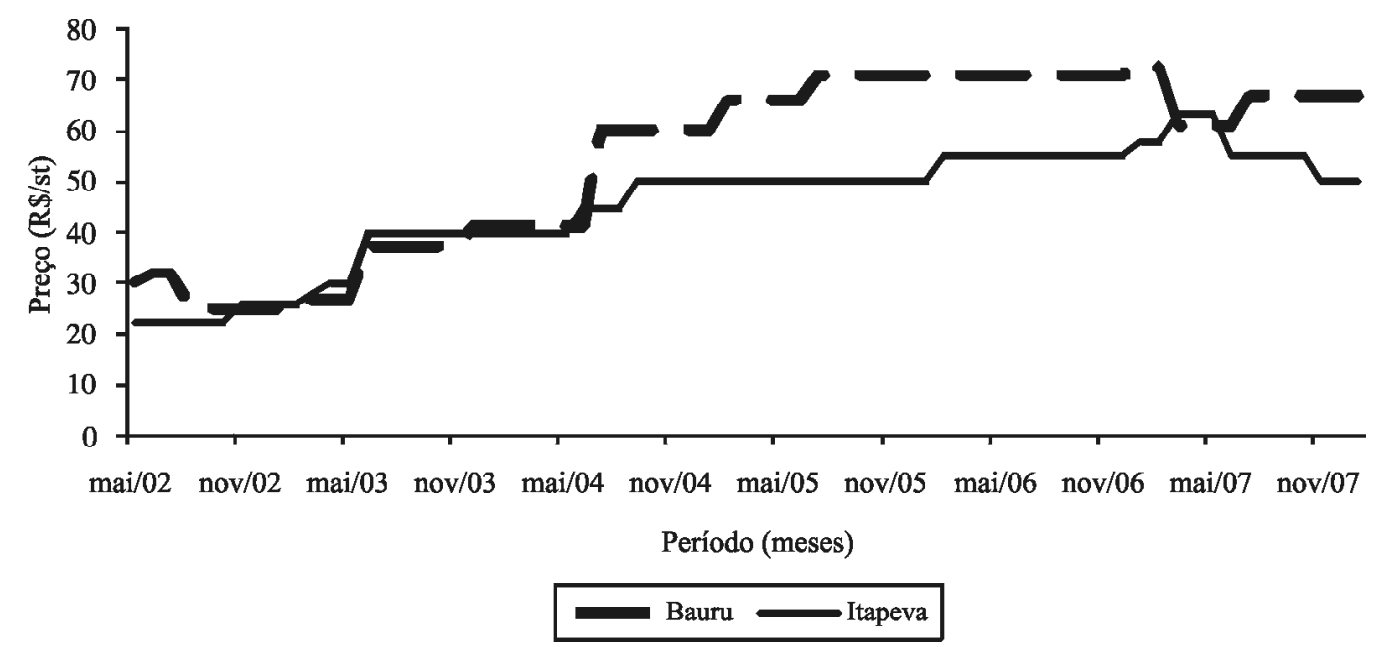

Figura 1 - Preço nominal da madeira de Eucalyptus spp. em Itapeva e Bauru, maio de 2002 a janeiro de 2008.

Figure 1 - Nominal price of Eucalyptus spp. wood in Itapeva and Bauru, from may 2002 to January 2008. 
Tabela 1 - Resultados do teste de ADF em nível e na primeira diferença para as séries mensais de preços da madeira de Eucalyptus spp., maio de 2002 a janeiro de 2007.

Table 1 - Results of ADF test in level and first difference for monthly prices series of Eucalyptus spp. wood, from May 2002 to January 2007.

\begin{tabular}{|c|c|c|c|c|c|}
\hline \multirow{2}{*}{ Praças } & \multirow{2}{*}{ Modelo } & \multicolumn{2}{|c|}{ Valores Críticos em nível de $1 \%$} & \multicolumn{2}{|c|}{ Valores Calculados do teste de ADF } \\
\hline & & Série em Nível & Série em $1^{\mathrm{a}}$ diferença & Série em Nível & Série em $1^{\mathrm{a}}$ diferença \\
\hline \multirow{3}{*}{ Itapeva } & $\begin{array}{l}\text { Com intercepto e } \\
\text { tendência }\end{array}$ & $-4,130526$ & $-4,133838$ & $-1,862178$ & $-7,942828$ \\
\hline & $\begin{array}{l}\text { Somente com } \\
\text { intercepto }\end{array}$ & $-3,552666$ & $-3,555023$ & $-1,296309$ & $-7,900617$ \\
\hline & $\begin{array}{l}\text { Sem intercepto e } \\
\text { sem tendência }\end{array}$ & $-2,606911$ & $-2,607686$ & 2,168958 & $-7,038967$ \\
\hline \multirow{3}{*}{ Bauru } & $\begin{array}{l}\text { Com intercepto e } \\
\text { tendência }\end{array}$ & $-4,130526$ & $-4,133838$ & $-2,046708$ & $-7,617716$ \\
\hline & $\begin{array}{l}\text { Somente com } \\
\text { intercepto }\end{array}$ & $-3,552666$ & $-3,555023$ & $-0,695888$ & $-7,692155$ \\
\hline & $\begin{array}{l}\text { Sem intercepto e } \\
\text { sem tendência }\end{array}$ & $-2,606911$ & $-2,607686$ & 1,460579 & $-7,340909$ \\
\hline
\end{tabular}

Fonte: Dados da pesquisa.

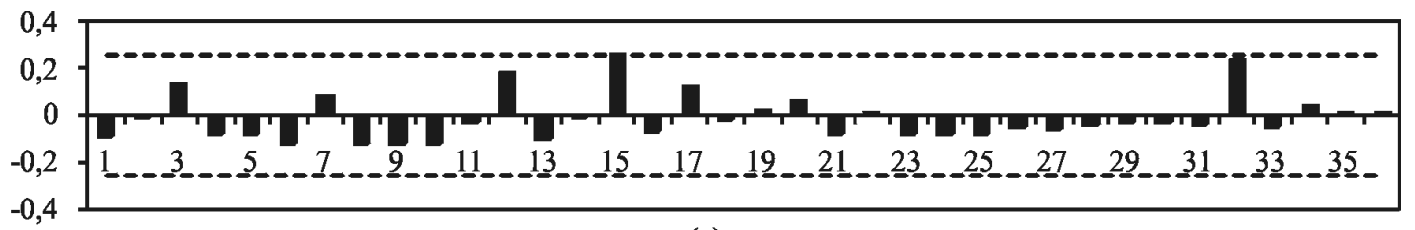

(a)

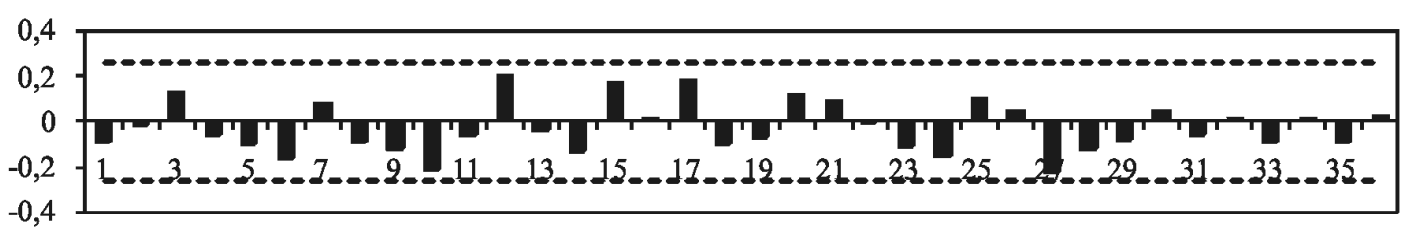

(b)

Fonte: Dados da Pesquisa.

Figura 2 - FAC (a) e FACP (b) em primeira diferença para as séries de preços da madeira em Itapeva, maio de 2002 a janeiro de 2007.

Figure 2 - Function of Autocorrelation $(A C F)(a)$ and Function of Partial Autocorrelation $(P A C)(b)$ in first difference for price series of wood in Itapeva, May 2002 to January 2007.

Entretanto, para a seleção dos modelos foram considerados a FAC e da FACP, o princípio de parcimônia, a significância dos coeficientes, o comportamento dos resíduos e os critérios de informação de Akaike (AIC) e Schwarz (SCH). Em outras palavras, foi selecionado o modelo que apresentou menor valor para os critérios $\mathrm{AIC}$ e SCH, resíduos com comportamento aleatório do tipo ruído branco e coeficientes significativos como mostrado a seguir.

ii) Estimação

Os resultados da estimação dos parâmetros do processo ARIMA para o preço da madeira de Eucalyptus spp. em Itapeva e Bauru estão na Tabela 2. Vários modelos foram estimados. Contudo, na Tabela 2, encontram-se apenas os que apresentaram coeficientes significativos.

Cerne, Lavras, v. 16, n. 1, p. 41-52, jan./mar. 2010 


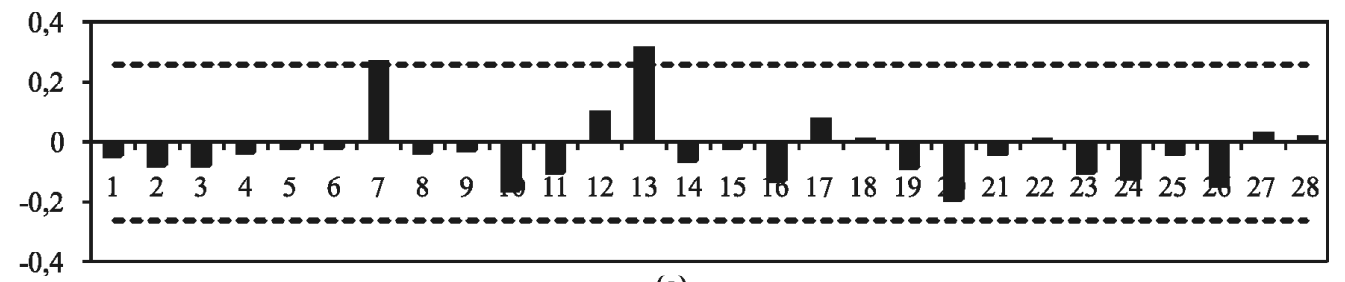

(a)

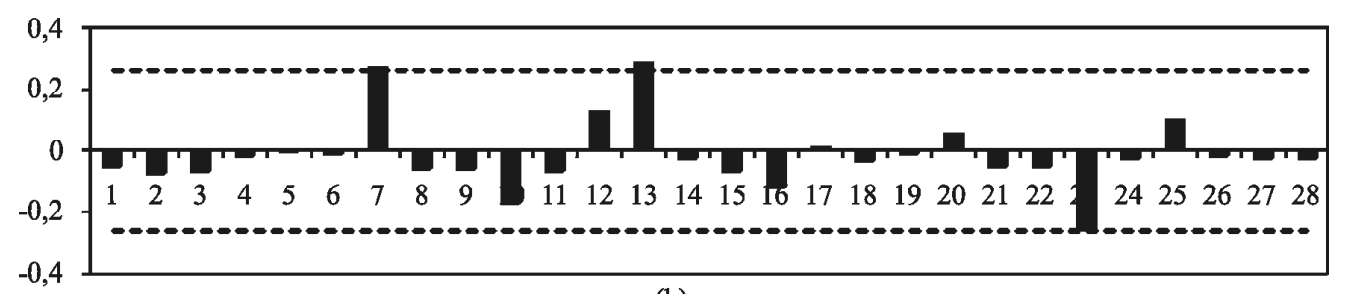

(b)

Fonte: Dados da Pesquisa.

Figura 3 - FAC (a) e FACP (b) em primeira diferença para as séries de preços da madeira em Bauru, maio de 2002 a janeiro de 2007.

Figure 3 - ACF (a) and PAC (b) of wood price series in Bauru, from May 2002 to January 2007.

Com base nos critérios de AIC e SCH, escolheu-se o modelo I - ARIMA $(0,1,4)$ para previsão do preço da madeira de Eucalyptus spp. em Itapeva.

No caso de Bauru, com base na FAC e na FACP, no princípio de parcimônia, na significância dos coeficientes e no comportamento dos resíduos o melhor modelo de previsão de preços da madeira de Eucalyptus spp. em Bauru seria o modelo II - ARIMA $(1,1,1)$. Todavia, observando o comportamento da FAC e na FACP, bem como a significância dos coeficientes, o comportamento dos resíduos e os critérios de informação de AIC e SCH, concluiu-se que o melhor modelo de previsão de preços seria o modelo I - ARIMA $(2,1,2)$ (Tabela 2).

Sintetizando, a equação estimada do modelo ARIMA, para Itapeva e para Bauru assume as formas apresentadas nas equações 17 e 18, respectivamente.

$$
\begin{aligned}
Y_{t}= & 0,018120+\varepsilon_{t}-0,242736 \varepsilon_{t-1}+0,312397 \varepsilon_{t-2}+0,469612 \varepsilon_{t-3} \\
& -0,524318 \varepsilon_{t-4} \\
W_{t}= & 0,013991-0,130276 W_{t-1}-0,873623 W_{t-2}+\varepsilon_{t}-0,982526 \varepsilon_{t-1} \\
& +0,994953 \varepsilon_{t-2}
\end{aligned}
$$

Assim como nos estudos sobre previsão de preços apresentados anteriormente para a área florestal, os modelos que proporcionaram melhor ajuste para a previsão de preços da madeira foram aqueles com os menores valores para p, d e q. Além disso, como no estudo de Coelho Junior et al. (2009), Silva \& Silva (1996) e Soares et al. (2008), não foram observados os modelos SARIMA. iii) Avaliação

$\mathrm{Na}$ avaliação dos modelos escolhidos, verificou-se que o comportamento médio dos seus resíduos foi satisfatório, obtendo resíduos próximos a um ruído branco conforme Figuras 4 e 5. Destarte, constata-se que os modelos mostraram-se satisfatórios para as estimações realizadas.

iv) Previsão

As previsões dos preços da madeira de Eucalyptus spp. em Itapeva e Bauru, no período de fevereiro de 2007 a janeiro de 2008, estão na Tabela 3.

A previsão feita pelo modelo I - ARIMA $(0,1,4)$ para Itapeva superestimou o preço da madeira de Eucalyptus spp. em 3,28\%, no período de fevereiro de 2007 a janeiro de 2008, sendo que os maiores desvios foram observados em junho e novembro de 2007 (Tabela 3).

A previsão feita pelo modelo I - ARIMA $(2,1,2)$ referente a Bauru superestimou o preço da madeira de Eucalyptus spp. em 1,91\%, no período de fevereiro de 2007 a janeiro de 2008, sendo que o maior desvio foi verificado em março de 2007 (Tabela 3).

O elevado desvio verificado em março de 2007 para Bauru e em junho e novembro de 2007 para Itapeva, ocorreu uma vez que o preço observado nesses períodos foi bem inferior ao preço previsto, quando comparado com os demais períodos considerados (Tabela 3). Todavia, isso não invalida os resultados, pois se deve considerar a análise como um todo e não apenas o erro (\%), isto é, deve ser considerada a significância dos coeficientes estimados

Cerne, Lavras, v. 16, n. 1, p. 41-52, jan./mar. 2010 
Tabela 2 - Modelos estimados para previsão do preço da madeira de Eucalyptus spp. em Itapeva e Bauru.

Table 2 - Estimated models for forecasting wood price of Eucalyptus spp. in Itapeva-SP and Bauru-SP.

\begin{tabular}{|c|c|c|c|c|c|c|c|}
\hline Praça & Modelo & Variáveis & Coeficiente & Erro-Padrão & Teste $\mathrm{t}$ & AIC & $\mathrm{SCH}$ \\
\hline \multirow{7}{*}{ Itapeva } & \multirow{5}{*}{$\begin{array}{c}\text { I } \\
\operatorname{ARIMA}(0,1,4)\end{array}$} & $\mathrm{C}$ & $0,018120 *$ & 0,006431 & 2,817588 & \multirow{5}{*}{$-3,17691$} & \multirow{5}{*}{$-2,99769$} \\
\hline & & $\theta_{1}$ & $-0,242736 * *$ & 0,123119 & $-1,971554$ & & \\
\hline & & $\theta_{2}$ & $0,312397 *$ & 0,101363 & 3,081968 & & \\
\hline & & $\theta_{3}$ & $0,469612 *$ & 0,105481 & 4,452089 & & \\
\hline & & $\theta_{4}$ & $-0,524318 *$ & 0,115423 & $-4,542564$ & & \\
\hline & \multirow{2}{*}{$\begin{array}{c}\text { II } \\
\operatorname{ARIMA}(0,1,1)\end{array}$} & $\mathrm{C}$ & $0,014301 *$ & 0,006182 & 2,313201 & \multirow[b]{2}{*}{$-4,27341$} & \multirow[b]{2}{*}{$-4,20172$} \\
\hline & & $\theta_{15}$ & $0,918822 *$ & 0,028266 & 32,50629 & & \\
\hline \multirow{14}{*}{ Bauru } & \multirow{5}{*}{$\begin{array}{c}\text { I } \\
\operatorname{ARIMA}(2,1,2)\end{array}$} & $\mathrm{C}$ & $0,013991^{\mathrm{ns}}$ & 0,009289 & 1,506188 & \multirow{5}{*}{$-2,44948$} & \multirow{5}{*}{$-2,27026$} \\
\hline & & $\phi_{1}$ & $0,130276 *$ & 0,040422 & 3,222908 & & \\
\hline & & $\phi_{2}$ & $-0,873623 *$ & 0,043540 & $-20,06493$ & & \\
\hline & & $\theta_{1}$ & $-0,185955 *$ & 0,042338 & $-4,392150$ & & \\
\hline & & $\theta_{2}$ & $0,994953 *$ & 0,019415 & 51,24784 & & \\
\hline & \multirow{3}{*}{$\begin{array}{c}\text { II } \\
\operatorname{ARIMA}(1,1,1)\end{array}$} & $\mathrm{C}$ & $0,020768 *$ & 0,008584 & 2,419341 & \multirow{3}{*}{$-2,85419$} & \multirow{3}{*}{$-2,74266$} \\
\hline & & $\phi_{7}$ & $-0,495337 *$ & 0,104315 & $-4,748468$ & & \\
\hline & & $\theta_{7}$ & $0,982526 *$ & 0,046230 & 21,25319 & & \\
\hline & \multirow{3}{*}{$\begin{array}{c}\text { III } \\
\operatorname{ARIMA}(1,1,1)\end{array}$} & $\mathrm{C}$ & $0,021069^{\mathrm{ns}}$ & 0,018759 & 1,123154 & \multirow{3}{*}{$-3,06895$} & \multirow{3}{*}{$-2,95742$} \\
\hline & & $\phi_{7}$ & $0,383032 *$ & 0,131726 & 2,907803 & & \\
\hline & & $\theta_{13}$ & $0,912214 *$ & 0,044358 & 20,56500 & & \\
\hline & \multirow{3}{*}{$\begin{array}{c}\text { IV } \\
\operatorname{ARIMA}(1,1,1)\end{array}$} & $\mathrm{C}$ & $0,003455 *$ & 0,019235 & 0,179641 & \multirow{3}{*}{$-3,41144$} & \multirow{3}{*}{$-3,29335$} \\
\hline & & $\phi_{13}$ & $0,409453 *$ & 0,079968 & 5,120206 & & \\
\hline & & $\theta_{7}$ & $0,944651 *$ & 0,020416 & 46,26925 & & \\
\hline
\end{tabular}

Fonte: Dados da pesquisa. *significativo em nível de $1 \%$ de probabilidade; $* *$ significativo em nível de $5 \%$ de probabilidade; sc $=$ sem correlação serial nos resíduos.

Cerne, Lavras, v. 16, n. 1, p. 41-52, jan./mar. 2010 


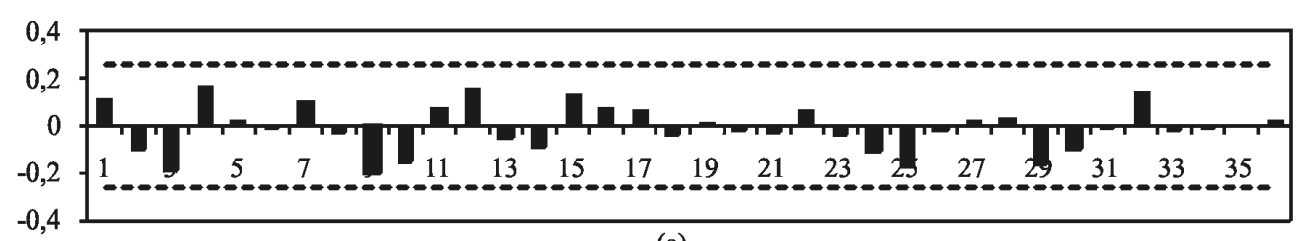

(a)

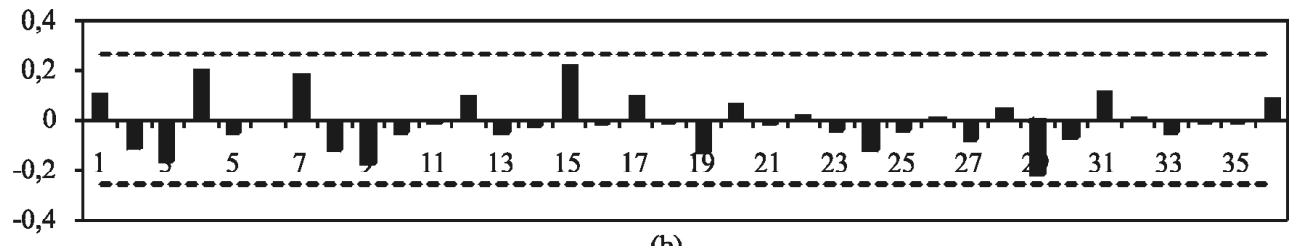

Fonte: Dados da Pesquisa.

(b)

Figura 4 - FAC (a) e FACP (b) dos resíduos do modelo de previsão de preços da madeira em Itapeva.

Figure 4-ACF (a) and PAC (b) of residuals of price forecast model of Eucalyptus spp. wood in Itapeva.

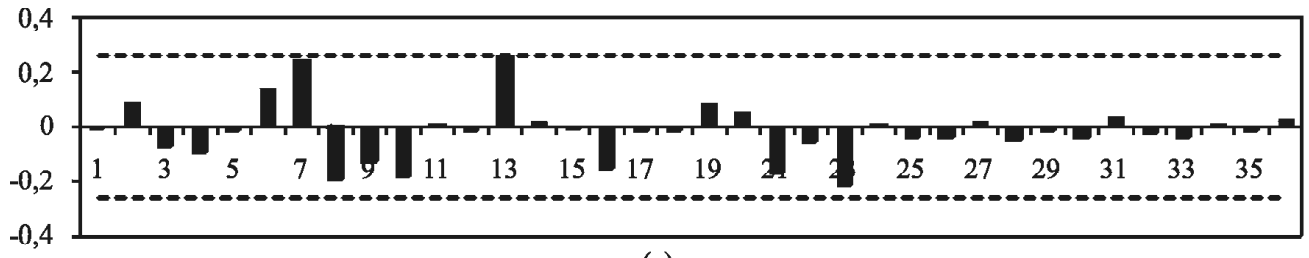

(a)

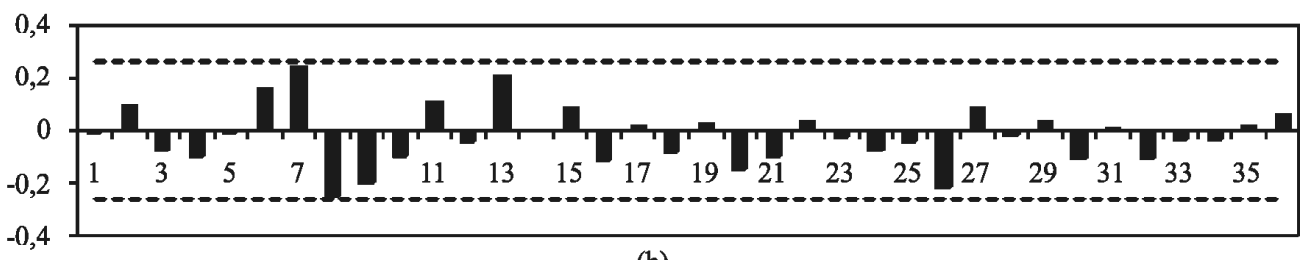

(b)

Fonte: Dados da Pesquisa.

Figura 5 - FAC (a) e FACP (b) dos resíduos do modelo de previsão de preços da madeira em Bauru.

Figure 5-ACF (a) and PAC (b) of resids of wood price forecast model of Eucalyptus spp. in Bauru.

do modelo escolhido, assim como o comportamento dos resíduos e a capacidade de previsão do modelo. Os resultados dessa última análise estão apresentados a seguir.

$\mathrm{Na}$ Tabela 4, encontram-se os componentes de desigualdade de Theil que são úteis para mostrar se o modelo de previsão é adequado. Mais precisamente, um modelo adequado é aquele cujo coeficiente de desigualdade de Theil apresente pequeno (próximo de zero) e os componentes de sua decomposição - proporção de tendenciosidade e proporção de variância sejam próximos de zero e a proporção de covariância seja próxima de um (SANTOS \& LIMA, 2006).
O CDT encontrado sugere um bom ajustamento do modelo e, consequentemente, das previsões para Itapeva e Bauru, pois é da ordem de 0,02 para as duas praças analisadas. Ressalta-se que este coeficiente varia entre zero e um, sendo que zero indica um ajuste perfeito. Quanto mais próximo da unidade, pior o ajustamento. Com relação à $\mathrm{PT}, \mathrm{PV}$ e $\mathrm{PC}$, o ideal é valor baixo para $\mathrm{PT}$ e PV e valor alto para PC, o que foi observado nos resultados obtidos para o preço da madeira de Eucalyptus spp. em Itapeva e Bauru (Tabela 4). Desse modo, pode-se dizer que os modelos de previsão foram adequados.

Cerne, Lavras, v. 16, n. 1, p. 41-52, jan./mar. 2010 
Tabela 3 - Previsão do preço da madeira de Eucalyptus spp. em Itapeva e Bauru, fevereiro de 2007 a janeiro de 2008.

Table 3 - Eucalyptus spp. wood price forecast in Itapeva and Bauru from February 2007 to January 2008.

\begin{tabular}{cccccccccccc}
\hline \multirow{2}{*}{ Período } & \multicolumn{2}{c}{ Valores Observados } & \multicolumn{2}{c}{ Previsão } & \multicolumn{2}{c}{ Limite Inferior } & \multicolumn{2}{c}{ Limite Superior } & \multicolumn{2}{c}{ Erro (\%) } \\
\cline { 2 - 8 } & Itapeva & Bauru & Itapeva & Bauru & Itapeva & Bauru & Itapeva & Bauru & Itapeva & Bauru \\
\hline fev/07 & 58 & 72 & 58,96 & 74,34 & 56,14 & 69,28 & 61,79 & 79,41 & 1,66 & 3,26 \\
mar/07 & 63 & 61 & 61,15 & 73,10 & 58,22 & 68,12 & 64,07 & 78,09 & $-2,94$ & 19,84 \\
abr/07 & 63 & 61 & 64,43 & 61,34 & 61,35 & 57,16 & 67,51 & 65,52 & 2,27 & 0,56 \\
mai/07 & 63 & 61 & 63,12 & 59,91 & 60,10 & 55,83 & 66,14 & 63,99 & 0,19 & $-1,79$ \\
jun/07 & 55 & 61 & 65,20 & 61,62 & 62,08 & 57,42 & 68,31 & 65,82 & 18,54 & 1,02 \\
jul/07 & 55 & 67 & 56,82 & 63,40 & 54,11 & 59,08 & 59,54 & 67,72 & 3,32 & $-5,38$ \\
ago/07 & 55 & 67 & 54,11 & 67,89 & 51,53 & 63,26 & 56,70 & 72,52 & $-1,61$ & 1,33 \\
set/07 & 55 & 67 & 51,03 & 66,71 & 48,59 & 62,16 & 53,47 & 71,26 & $-7,21$ & $-0,44$ \\
out/07 & 55 & 67 & 59,51 & 67,33 & 56,67 & 62,74 & 62,36 & 71,91 & 8,21 & 0,49 \\
nov/07 & 50 & 67 & 59,90 & 68,63 & 57,04 & 63,95 & 62,77 & 73,31 & 19,80 & 2,43 \\
dez/07 & 50 & 67 & 53,30 & 68,23 & 50,75 & 63,58 & 55,85 & 72,88 & 6,61 & 1,84 \\
jan/08 & 50 & 67 & 45,28 & 66,87 & 43,12 & 62,32 & 47,45 & 71,43 & $-9,43$ & $-0,19$ \\
\hline
\end{tabular}

Fonte: Dados da pesquisa.

Tabela 4 - Resultados da avaliação da previsão do preço da madeira de Eucalyptus spp. em Bauru, fevereiro de 2007 a janeiro de 2008.

Table 4-Results of the evaluation of wood price of Eucalyptus spp. forecast in Bauru, February 2007 to January 2008.

\begin{tabular}{lcc}
\hline \multicolumn{1}{c}{ Testes } & Bauru & Itapeva \\
\hline Raiz do erro de previsão quadrático médio (REPQM) & 3,039159 & 2,747218 \\
Erro absoluto médio (EAM) & 1,653003 & 1,9787245 \\
Percentual do erro absoluto médio (PEAM) & 3,532271 & 4,451681 \\
Coeficiente de Desigualdade de Theil (CDT) & 0,026625 & 0,029219 \\
$\quad$ Proporção de tendenciosidade (PT) & 0,000799 & 0,023561 \\
Proporção de variância (PV) & 0,010562 & 0,059028 \\
Proporção de covariância (PC) & 0,988638 & 0,917410 \\
\hline
\end{tabular}

Fonte: Dados da pesquisa.

\section{CONCLUSÕES}

Com base nos resultados obtidos, concluiu-se que o modelo I - ARIMA $(0,1,4)$ e o modelo II - ARIMA $(2,1,2)$ referentes a Itapeva e Bauru, respectivamente, foram eficientes e apresentaram projeções satisfatórias para previsão dos preços da madeira de Eucalyptus spp. nessas regiões, no período de fevereiro de 2007 a janeiro de 2008, pois foram observados resíduos próximos a um ruído branco, erro (\%) médio baixo, capacidade de previsão adequada e elevada significância dos coeficientes estimados; e os modelos SARIMA não foram identificados, pois não foram constatados efeito de sazonalidade.

Ressalta-se que a principal limitação do trabalho foi a inexistência de uma série de preços da madeira mais longa para Itapeva (SP) e Bauru (SP).

\section{AGRADECIMENTOS}

Os autores agradecem ao Conselho Nacional de Desenvolvimento Científico e Tecnológico (CNPq), à Fundação de Amparo à Pesquisa do Estado de Minas Gerais (FAPEMIG) e à Universidade Federal de Viçosa. 


\section{REFERÊNCIAS BIBLIOGRÁFICAS}

ASSOCIAÇÃO BRASILEIRA DE CELULOSE E PAPEL. Relatório estatístico florestal. São Paulo, 2006. Disponível em: $\overline{\mathrm{h}} \mathrm{ttp}: / / \mathrm{w} w \mathrm{w}$ ipef.br/estatisticas/relatorios/Bracelpa」 'Relatorio_Estatistico_Florestal-2006-ĒNG fev. 2008.

ASSOCIAÇÃO BRASILEIRA DE CELULOSE E PAPEL. Setor de celulose e papel. São Paulo, 2007. Disponível em: <http:// www.bracelpa org.br/bra/eveventos bracelpat ipress_release.pdfy. Acesso em: 3 abr. 2008.

ASSOCIAÇÃO BRASILEIRA DE PRODUTORES DE FLORESTAS PLANTADAS. Anuário estatístico da ABRAF. Brasília, 2008. CD-ROM.

ASSOCIAÇÃO MINEIRA DE SILVICULTURA. Eucalyptus spp. Disponível em: Khttp://Www.silviminas.com.brì. Acesso em: 20 mar. 2008.

BASU, J. P. Cointegration and market integration: an application to the potato markets in rural West Bengal, India. 2006. Disponível em! ${ }^{-}$http://agecon.lib.umn.edu/cgi-bin ipdf view.pl?paperid=22101\&ftype=pdfy. Acesso em: 28 mar. 2008.

BOX, G. E. P.; JENKINS, G. M. Times series analysis: forecasting and control. São Francisco: Holden-Day, 1976.

CENTRO DE ESTUDOS AVANÇADOS EM ECONOMIA APLICADA. Informativo CEPEA setor florestal. Disponível em: ‘http://www.cepea.esalg.usp.brì Acesso em: 10 jun. 2008.

CÉZAR, S. A. G.; SUEYOSHI, M. L. S.; PINO, F. A.; FRANCISCO, V. L. F. S.; AMARAL, A. M. P. Ajustamento sazonal de preços com inflação. Agricultura em São Paulo, São Paulo, v. 42, n. 2, p. 39-63, 1995.

COELHO JUNIOR, L. M.; REZENDE, J. L. P.; BORGES, L. A. C.; OLIVEIRA, A. D. Análise temporal da Borracha Natural Brasileira. Cerne, Lavras, v. 15, n. 1, p. 19-26, jan./mar. 2009.

COELHO JUNIOR, L. M.; REZENDE, J. L. P. de; CALEGARIO, N.; SILVA, M. L. da. Análise longitudinal dos preços do carvão vegetal, no Estado de Minas Gerais. Revista Árvore, Viçosa, v. 30, n. 3, p. 429-438, maio/jun. 2006 a.
COELHO JUNIOR, L. M.; REZENDE, J. L. P.; SÁFADI, T.; CALEGARIO, N. Análise temporal do preço do carvão vegetal oriundo de floresta nativa e de floresta plantada. Scientia Forestalis, Piracicaba, n. 70, p. 39-48, abr. 2006b.

ENDERS, W. Applied econometric time series. New York: J. Wiley, 1995. $433 \mathrm{p}$.

FISHER, S. Séries univariantes no tempo: metodologia de Box e Jenkins. Porto Alegre: Fundação de Economia e Estatística, 1982.

GOMES, M. T. M. Potencialidades de inserção do carvão vegetal em bolsa de mercadorias. 2006. 71 f. Dissertação (Mestrado em Ciência Florestal) - Universidade Federal de Viçosa, Viçosa, 2006.

GUJARATI, D. N. Econometria básica. 3. ed. São Paulo: Makron Books, 2000. 846 p.

OLIVEIRA, R. A.; BUONGIORNO, J.; KMIOTEK, A. M. Time series forecasting models of lumber cash, futures, and basis prices. Forest Science, Lawrence, v. 23, n. 2, p. 269-279, 1977.

ROCHA, F. G. Contribuição de modelos de séries temporais para a previsão da arrecadação de ISS. 2003. 123 f. Dissertação (Mestrado em Economia) - Universidade Estadual de Campinas, Campinas, 2003.

SANTOS, C. M. dos; LIMA, J. E. Análise de previsão da arrecadação do ICMS no Estado de Minas Gerais. Revista de Economia e Administração, São Paulo, v. 5, n. 4, p. 413-423, out./dez. 2006.

SEDDIGHI, H. R.; LAWYER, K. A.; KATOS, A. V. Econometrics: a pratical approach. London: Routledge, 2000. $396 \mathrm{p}$.

SILVA, M. L. da; OLIVEIRA, R. J. de; VALVERDE, S. R.; MACHADO, C. C.; PIRES, V. A. V. Análise do custo e do raio econômico de transporte de madeira de reflorestamentos para diferentes tipos de veículos. Revista Árvore, Viçosa, v. 31, n. 6, p. 1073-1079, 2007.

SILVA, M. L. da; SILVA, J. M. A. da. Análise do comportamento temporal dos preços do carvão vegetal: aplicação e avaliação da metodologia "Box e Jenkins". Revista Árvore, Viçosa, v. 20, n. 1, p. 57-67, 1996.

Cerne, Lavras, v. 16, n. 1, p. 41-52, jan./mar. 2010 
SOARES, N. S. Potencial de implantação de um contrato futuro da madeira de reflorestamento. 2006. $121 \mathrm{f}$. Dissertação (Mestrado em Ciência Florestal) - Universidade Federal de Viçosa, Viçosa, 2006.

SOARES, N. S.; SILVA, M. L. da; LIMA, J. E. de; CORDEIRO, S. A. Análise de previsões do preço da borracha natural no Brasil. Scientia Forestalis, Piracicaba, v. 36, n. 80, p. 285-294, dez. 2008.

SONG, N. Structural and forecasting softwood lumber models with a time series approach. 2003. Disponível em: $\langle\bar{h}$ ttp://etd.1su.edu/docs/available/etd-071 $25006-09325 \overline{1}$, unrestricted/Song_dis.pdff $\rangle^{\prime}$. Acesso em: 15 jan. 2008.

VALVERDE, S. R.; SOARES, N. S.; SILVA, M. L. da; JACOVINE, L. A. G.; NEIVA, S. A. Reflexões sobre o Mercado da Madeira de Eucalyptus spp. no Brasil. Revista da Madeira, Viçosa, v. 15, n. 87, fev. 2005.

VOTORANTIM CELULOSE E PAPEL. Sustainable growth with value creation and solid returns. São Paulo, 2004. 28 p.

Cerne, Lavras, v. 16, n. 1, p. 41-52, jan./mar. 2010 